\begin{tabular}{|c|c|}
\hline 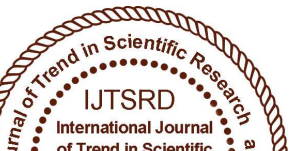 & $\begin{array}{l}\text { International Journal of Trend in Scientific } \\
\text { Research and Development (IJTSRD) }\end{array}$ \\
\hline 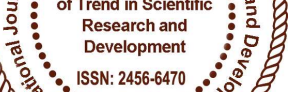 & International Open Access Journal \\
\hline 000 & ISSN No: 2456 - 6470 | www.ijtsrd.com | Volume - 2 | Issue - 3 \\
\hline
\end{tabular}

\title{
Design of Blood Monitor Sensor Model to Determine Glucose Level in Blood Sample
}

\author{
Dr. Sarita. B. Dhoble, Ms. Sheetal D. Bhoyar, Mr. Prafulla N. Aerkewar \\ Assistant Professor, Department of Electronics Engineering, \\ Priyadarshini Bhagwati College of Engineering, \\ Nagpur, Maharashtra, India
}

\begin{abstract}
The prime intention of this research work has been to study and develop glucose monitoring system using IR sensor and ultrasonic MEMS to determine the blood density and blood pressure in patient blood sample. It is needless to emphasize the great importance of glucose level testing in diabetes life. Blood is combination of that high content it may like proteins, DNA, RNA, plasma etc. This research presents a spectroscopic sensor model to study and analyze the blood level and its parameter of sample blood before treatment. In this research work, compare the predicted glucose values with those from NIR spectroscopy using various glucose monitoring techniques. The predicted result being used is various patient monitoring system.
\end{abstract}

Keywords: Blood, glucose, sensor, invasive method, non-invasive

\section{INTRODUCTION}

According to World Health Organization (WHO), the pervasiveness of diabetes was estimated to be $9 \%$ among adults, 4.9 million deaths were caused by diabetes in 2014 and diabetes will be the 7th leading disease cause of death in 2030. Diabetes and its complications are responsible for early death, where 1 person dies in every 7 seconds. With regards to economics, cost of diabetes covers 6 to $15 \%$ of the budget of National Health System in the European Union[1].

Glucose is the main source of energy for the human body. Glucose levels are regulated to keep the body

homeostasis so that blood glucose level remains stable and relatively constant. There are many hormones that are involved in this process but insulin is the most important one. Insulin is produced by the beta cells of the pancreas and it is provided to remove excess glucose from the blood.

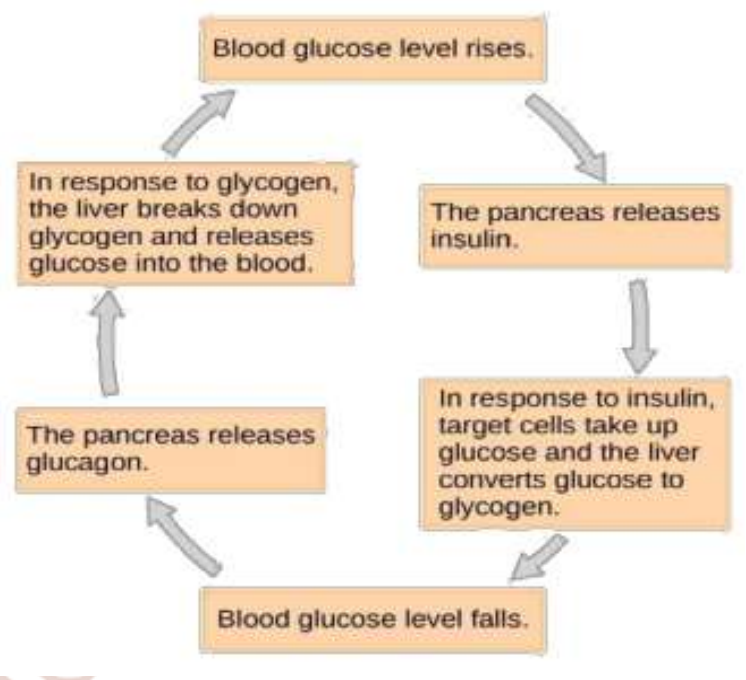

Fig1.:Regulation of Blood Glucose Levels by Insulin and Glucagon

\section{A. Types of Diabetes :}

The three most common type of diabetes are

1. Type 1 diabetes,

2. Type 2 diabetes,

3. Gestational diabetes. 
Although there are also other types of diabetes including congenital diabetes, cystic-fibrosis related diabetes, and steroid diabetes. Type 1 or Insulin Dependent Diabetes Mellitus (IDDM) is an auto immune disease in which body cannot produce sufficient insulin leading to insulin deficiency due to loss of insulin- producing beta cells. Type 2 diabetes or Non-Insulin Dependent Diabetes Mellitus (NIDDM) is due to reduced insulin secretion. Gestational diabetes usually occurs to women during their pregnancy. It generally resolves after pregnancy or else it may lead to Type 2 diabetes. $90 \%$ cases of diabetes are of Type 2 diabetes which mostly affects the adult people.

\section{B. Complications related to Diabetes :}

Blood glucose level is the amount of glucose present in blood. It is commonly measured in $\mathrm{mg} / \mathrm{dl}$. Diabetes patients have abnormally excessive glucose level or diminished glucose level. Symptoms of diabetes include Polydipsia, Polyphagia, etc. as shown in Figure 2. This condition can be classified as hypoglycemic, where blood glucose level is less than $72 \mathrm{mg} / \mathrm{dl}$ or hyperglycemic, where blood glucose level is more than $200 \mathrm{mg} / \mathrm{dl}$. Hypoglycemia has short term effects and it usually affects brain. It is classified on the basis of glucose level:

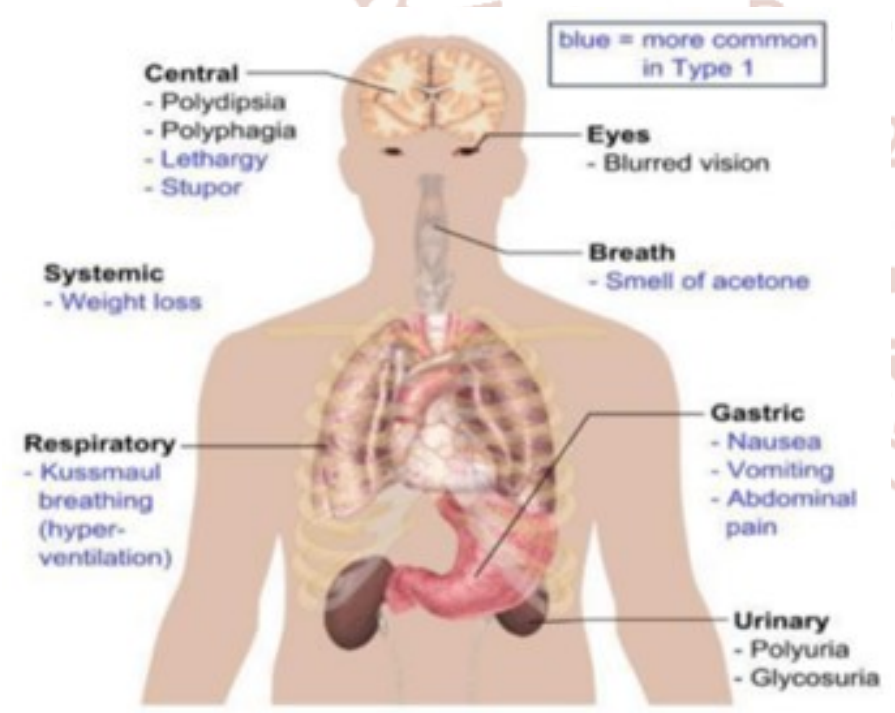

Figure 2. Symptoms of Diabetes

1. Mild hypoglycemia: Blood glucose level is between 55 and $70 \mathrm{mg} / \mathrm{dl}$. In this case, eating small amount of carbohydrate can restore normal glucose levels.
2. Moderate hypoglycemia: Blood glucose level is between 40and $55 \mathrm{mg} / \mathrm{dl}$. It is characterized by mood changes, confusion, blurred vision, weakness and drowsiness since it affects the central nervous system.

3. Severe hypoglycemia: Blood glucose level is less than $40 \mathrm{mg} / \mathrm{dl}$. It is characterized by convulsions, loss of consciousness and coma. In this case glucagon injection is required.

\section{Blood Glucose Level Monitoring}

Long term and short term complications can be reduced through proper diet, physical exercise, and medication. But to know the pattern of glucose changes of a diabetic patient, concentration of glucose in blood (glycaemia) needs to be monitored by using invasive and non-invasive glucose monitoring techniqe.
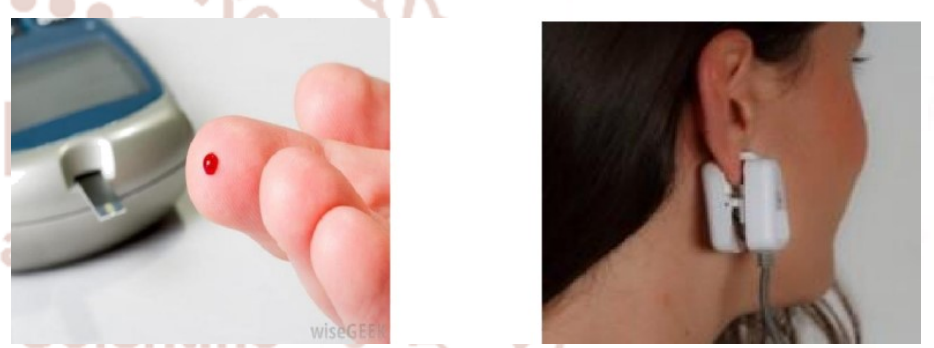

Figure 3. Invasive (Left) and Noninvasive (Right) Glucose Monitoring

\section{Proposed Noninvasive Blood Glucose Level Monitoring System :}

A. Proposed Method :

Proposed MEMS Sensor are used for monitoring the glucose concentration by using noninvasive approach. IR sensor and MEMS sensor work as a transmitter and receiver at operating point. Proposed model work on the principle of spectroscopy operating technique. Calibration system used to predict glucose concentration in input testing sample.

\section{B. Block Diagram of the Proposed Method :}

The proposed system with IR and ultrasonic MEMS sensors is illustrated in Figure 3.

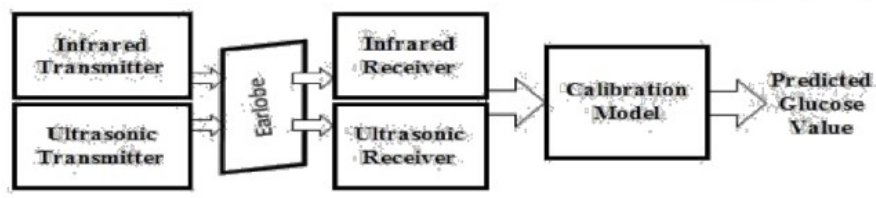

Figure 3. Block diagram of proposed NIBGM system 
Ultrasonic wave from ultrasonic MEMS sensor generates pressure wave in medium (such earlobe) and is collected by ultrasonic MEMS receiver. In order to generate ultrasonic wave, piezoelectric device is used. All the individual parts in that block diagram are explained below as follows:

1. Infrared Transmitter: The functional wavelength of $2270 \mathrm{~nm}$ will be utilized here for the experimental purposes. $\mathrm{T}$

2.Ultrasonic Transmitter: A piezoelectric based ultrasound transmitter with $2 \mathrm{MHz}$ of operating frequency will be supplied for the generation of acoustic wave. This unit is also connected to the same side of earlobe as Infrared Transmitter.

3. Infrared Receiver: The blood glucose tends to have a precise vibrational pattern for its respective concentration measurements tend to change in absorption will be responded by this very sensitive IR receiver unit.

4. Ultrasonic Receiver: Again, the piezoelectric based ultrasound receiver with $2 \mathrm{MHz}$ as operating frequency has been utilized here. The prime task of this unit is to plaid the pattern and orientations of the generated acoustic waves.

5. Calibration Model: The output values form two receivers will be fed to this model. Using multiple linear regression analysis, the values are processed in MATLAB to predict the blood glucose level concentration.

\section{Working Principle of the Proposed Method:}

Ultrasonic wave is a sound wave transmitted at a frequency greater than $20 \mathrm{KHz}$ or beyond the normal hearing range of human. When ultrasonic wave will pass through the biological tissue, it produces vibrational patterns into the medium. These wave can be generated from a piezoelectric transducer. This leads to the generation of acoustic energy due to its existence in the ultrasonic field.

Hence we will be able to monitor the glucose level using the pressure Measurement This is better can be explained by Beer-Lamberts law as shown in given equation.
$A(v)=-\log I(v) / I$ o(v)

Where,

$\mathrm{A}(\mathrm{v})=$ absorbance at wavelength of $1 / \mathrm{v}$.

$\mathrm{v}=$ wave number.

$\mathrm{I}=$ light intensity of the adjacent medium.

I $0=$ light intensity after penetrating through the medium.

Absorbance can also defined in terms of absorptivity coefficient, concentration of molecule and path length as shown in given equation .

$A(v)=a * b * c$

Where

$a=$ absorptivity coefficient

$\mathrm{b}=$ path length

$\mathrm{c}=$ concentration

Here, $\mathrm{c}$ is considered as the concentration of blood glucose. So, increase in level of glucose will increase the absorbance. In this way finding the value of glucose level is achieved by comparing absorbance value to a reference absorbance value.

\section{Results And Evaluation :}

In this paper, we present the impact of piezoelectric materials on pressure followed by pressure generated in blood and predicted blood glucose concentration. Then we compare the predicted glucose values with those from NIR spectroscopy using various glucose monitoring techniques.

\section{A. Noninvasive Monitoring of Blood Glucose Level}

Noninvasive method collect the data base by IR sensor and ultrasonic MEMS sensor and provide this data to calibration system to analyze glucose level.

\section{Simulation of Different Piezoelectric Materials}

In the analysis, for simulation purpose,blood density is considered as a basic parameters. The level of blood glucose is calculated by analyzing the value of blood density.Blood pressure is one of the prime objective to study the level of blood sample. 


\section{Using Ultrasonic MEMS Sensor}

Piezoelectric material is one of the best alternative to check pressure in patient blood sample. Alternatly this is used to calculate density of blood.As level of glocose concentration is increases, it used to increase blood density and then pressure gradient is calculated.

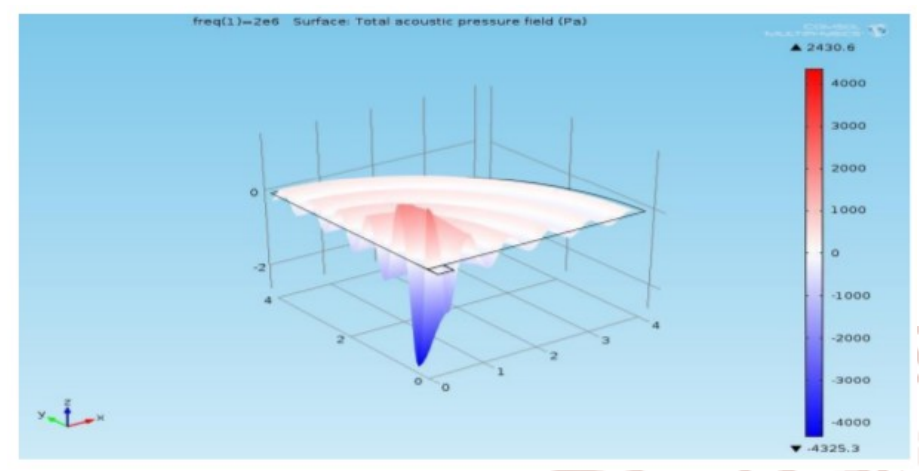

Figure 4 (a). Waveform of Acoustic PressureGenerated in Blood of Concentration $54.05 \mathrm{mg} / \mathrm{dL}$

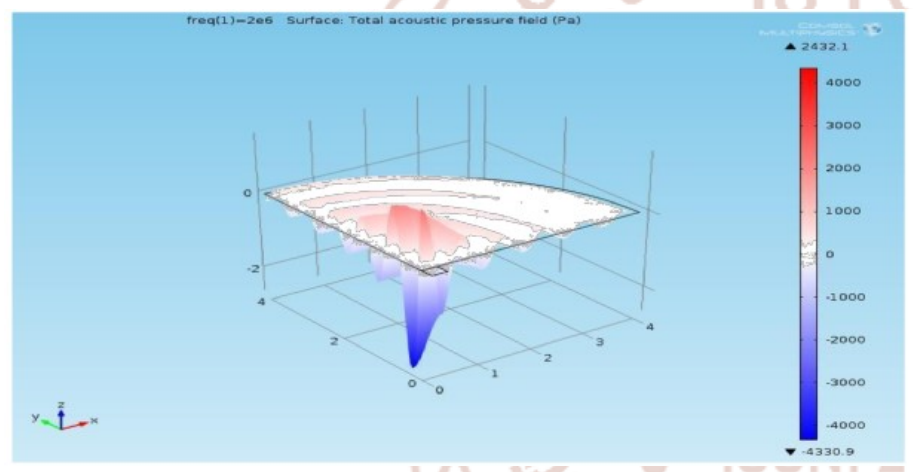

Figure 4 (b). Waveform of Acoustic Pressure Generated in Blood of Concentration $180.18 \mathrm{mg} / \mathrm{dL}$

\section{Conclusions :}

Both long term and short term complications from diabetes can be reduced through suggest diet, physical exercise, and proper medication. In order to determine the appropriate drugs, blood glucose concentration is regularly monitored. However, traditional invasive blood glucose monitoring systems are expensive, inconvenience, and painful (due to pricking fingers).

Proposed method is used to direct patient pressure gradient. It is used to monitor patient blood glucose level by calculating blood glucose density.

\section{References :}

1) World Health Organization. Diabetes. Media centreWorld Health Organization,2015. [online].Available from: http://www.who.int/mediacentre/factsheets/fs312/ en/. Accessed March 28, 2015.

2) International Diabetes Federation. Diabetes Atlas.IDF Diabetes Atlas, 2015 sixth edition. [online]. Available from: http://www.idf.org/diabetesatlas/. Accessed March 28, 2015.

3) Boundless. "Hormonal Regulation of Metabolism." Boundless Biology. Boundless, 03 Jul. 2014. [online]. Available from: https://www.boundless.com/biology/textbooks/bo undless-biology-textbook/the- endocrine-system37/regulation-of-body-processes-212/hormonalregulation-of- metabolism-799-12035/. Accessed March 28, 2015.

4) World Health Organization. About diabetes. Diabetes Programme. World Health Organization; 2015. Analine]. [online Availa http://www.who.int/diabetes/action_online/basics/ en/index.htmlAccessed March 28, 2015.

5) A. Ernest, "Diabetes mellitus and its complications : molecular mechanisms, epidemiology, and clinical medicine," New York Academy of Science, 2006,vol. Xiv, pp.532.

6) - Main symptoms of diabetes. [Online]. Available: http://commons.wikimedia.org/wiki/File:Main_sy mptoms_of_diabetes.svg. Accessed March 28, 2015.

7) S.N. Davis and G. Lastra-Gonzalez, "Diabetes and Low Blood Sugar (Hypoglycemia)," Journal of Clinical Endocrinology \& Metabolism, 2008, vol. 8 pp. 93.

8) Chi-Fuk So, Kup-Sze Choi, Thomas KS Wong, and Joanne WY Chung, "Recent advances in noninvasive glucose monitoring," Medical Devices: Evidence and Research, June 27, 2007.

9) Invasive Glucose Meter. [online]. Available: http://www.wisegeek.com/how-do-i- choose-thebest-non-invasive-glucose-meter.htm. Accessed March 28, 2015. 